\title{
Structural stability and electronic properties of low-index surfaces of SnS
}

\section{Citation}

Tritsaris, Georgios A., Brad D. Malone, and Efthimios Kaxiras. 2014. "Structural Stability and Electronic Properties of Low-Index Surfaces of SnS." Journal of Applied Physics 115 (17): 173702. https://doi.org/10.1063/1.4874775.

\section{Permanent link}

http://nrs.harvard.edu/urn-3:HUL.InstRepos:41384034

\section{Terms of Use}

This article was downloaded from Harvard University's DASH repository, and is made available under the terms and conditions applicable to Other Posted Material, as set forth at http:// nrs.harvard.edu/urn-3:HUL.InstRepos:dash.current.terms-of-use\#LAA

\section{Share Your Story}

The Harvard community has made this article openly available.

Please share how this access benefits you. Submit a story.

Accessibility 


\title{
Structural stability and electronic properties of low-index surfaces of SnS
}

\author{
Georgios A. Tritsaris, ${ }^{1}$ Brad D. Malone,${ }^{1}$ and Efthimios Kaxiras ${ }^{1,2, a)}$ \\ ${ }^{1}$ School of Engineering and Applied Sciences, Harvard University, Cambridge, Massachusetts 02138, USA \\ ${ }^{2}$ Department of Physics, Harvard University, Cambridge, Massachusetts 02138, USA
}

(Received 2 April 2014; accepted 22 April 2014; published online 2 May 2014)

\begin{abstract}
Thin film photovoltaic cells are increasingly important for cost-effective solar energy harvesting. Layered $\mathrm{SnS}$ is a promising absorber material due to its high optical absorption in the visible and good doping characteristics. We use first-principles calculations based on density functional theory to study structures of low-index surfaces of $\mathrm{SnS}$ using stoichiometric and oxygen-containing structural models, in order to elucidate their possible effect on the efficiency of the photovoltaic device. We find that the surface energy is minimized for the surface with orientation parallel to the layer stacking direction. Compared to stoichiometric surfaces, the oxygen-containing surfaces exhibit fewer electronic states near the band gap. This reduction of near-gap surface states by oxygen should reduce recombination losses at grain boundaries and interfaces of the $\mathrm{SnS}$ absorber, and should be beneficial to the efficiency of the solar cell. (C) 2014 AIP Publishing LLC.
\end{abstract}

[http://dx.doi.org/10.1063/1.4874775]

\section{INTRODUCTION}

Thin film photovoltaic (PV) cells are increasingly important for cost-effective conversion of sunlight into electricity. ${ }^{1,2}$ High conversion efficiencies $(>15 \%)$ have been achieved using absorber layers based on $\mathrm{CuInGaSe}_{2}$ (CIGS) or $\mathrm{CdTe},{ }^{3}$ but many elements in these systems are toxic and scarce. Layered tin monosulfide $(\mathrm{SnS})$ is a non-toxic material with high absorption in the visible region of the solar spectrum (absorption coefficient $>10^{4} \mathrm{~cm}^{-1}$ ). ${ }^{4-7}$ Conversion efficiencies of PV cells based on SnS are currently around 2\%, leaving much room for improvement. Indeed, experimental work has been achieving steady gains in the efficiency, ${ }^{8-12}$ but the properties of $\mathrm{SnS}$ samples depend on the synthesis route which makes it difficult to identify the structural factors responsible for these gains. For instance, Sinsermsuksakul et al. ${ }^{8}$ reported a shift in preferred crystallographic orientation of the $\mathrm{SnS}$ thin film during atomic layer deposition: the crystal plane perpendicular to the layer stacking direction changes from being parallel to being perpendicular to the substrate, as the film thickness increases. A change in preferred crystallographic orientation of SnS samples, prepared by RF sputtering, was also observed by Hartman et al. ${ }^{9}$ but in those studies the planes perpendicular to the layer stacking direction dominate in all samples irrespective of film thickness. The growth orientation of the film can affect the performance of the absorber layer because $\mathrm{SnS}$ is highly anisotropic. The optical and electronic properties depend on a number of features, including film thickness, grain size and grain orientation as well as on preparation conditions such as annealing temperature and atmosphere. The conversion efficiency of the PV cell also depends sensitively on the properties of the interface between the absorber and the buffer layers. ${ }^{13-16}$

Theory and atomistic simulation can elucidate how specific atomic-scale structural features of the absorber material

${ }^{\text {a)} E-m a i l: ~ k a x i r a s @ p h y s i c s . h a r v a r d . e d u ~}$ affect the efficiency of the PV cell, ${ }^{10,16-19}$ and in so doing, assist in the design of improved materials for efficient solar energy harvesting. In previous work, we investigated how the properties of few-layer and bulk SnS depend on the crystallographic orientation. ${ }^{18}$ Here, we use first-principles calculations based on density functional theory to investigate the atomic and electronic structure of the low-index surfaces of layered SnS. Specifically, we evaluate their relative stability by calculating surface energies and we discuss trends in electronic density of states associated with stoichiometric model surfaces as well as oxygen-containing structures which are expected to form during annealing in oxygen atmosphere. We find that an atom-thick layer of oxidized Sn eliminates surface states from the energy range near the band gap. This effect should reduce carrier recombination at grain boundaries in the SnS film and at the interface between the absorber and buffer layers in the PV cell. These findings provide useful guidelines for systematically improving the performance of PV cells based on SnS absorbers by controlled growth of the SnS thin film.

\section{MODELS AND METHODS}

$\mathrm{SnS}$ is an orthorhombic crystal consisting of weakly coupled bilayers of covalently bonded (within each bilayer) $\mathrm{Sn}-\mathrm{S}$ units. ${ }^{20}$ We used a unit cell with $4 \mathrm{Sn}$ and $4 \mathrm{~S}$ atoms to model the bulk structure of SnS, which is in the crystallographic space group Pnma, and extended slabs to model its low-index surfaces. The bilayers in $\mathrm{SnS}$ are arranged perpendicular to the $a$-axis. Each slab, modeling a different surface orientation, was constructed by truncation of the bulk structure of SnS along one of the six low-index crystallographic planes (Fig. 1). The minimal unit cell was repeated 3 times in the direction perpendicular to the surface for slabs modeling the [100] and [110] surface orientations and 4 times for the rest of the slabs, leading to 6 or more atomic layers thick structural models. The obtained unit cell was augmented by a void region with width $8 \AA$ on each side of the slab to 


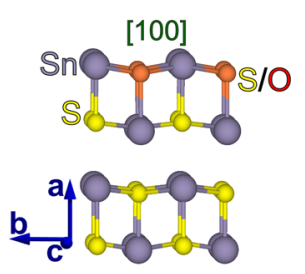

[110]

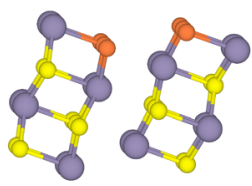

[010]

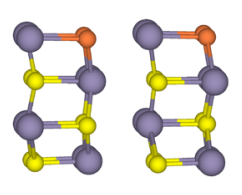

[101]

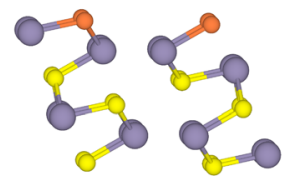

[001]

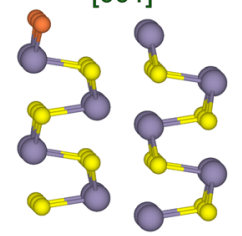

[011]

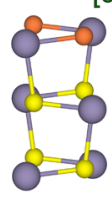

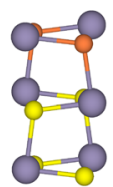

FIG. 1. Structural models of the low-index surfaces of layered tin monosulfide (SnS). Purple and yellow spheres represent Sn and S atoms, respectively. The surface sites colored orange are occupied by $\mathrm{S}$ atoms in the stoichiometric surfaces, or by oxygen atoms. Only the topmost atomic layers are shown for each orientation.

ensure that the wave functions vanish smoothly away from the surface along the non-periodic direction, as would be required in a model of the isolated system. The calculated surface energies are converged to within $0.01 \mathrm{~J} / \mathrm{m}^{2}$ for slabs. Oxygen-containing structures of $\mathrm{SnS}$ were modeled by substitution of all exposed $\mathrm{S}$ atoms by $\mathrm{O}$ atoms (orange spheres in Fig. 1). The positions of all atoms, except for those in the one or two planes at the center of the slab, were relaxed while keeping the lateral dimensions of the unit cell fixed to the corresponding bulk values. In the present work, we do not consider reconstructions that change the size of the surface unit cell, that is, we use supercells with $(1 \times 1)$ periodicity relative to the bulk-terminated plane. For the [001] surface orientation, we took into account rebonding of the surface $\mathrm{S}$ atoms in one $\mathrm{SnS}$ bilayer to the surface $\mathrm{Sn}$ atoms in the neighboring bilayer (Fig. 1), because we found that this local rearrangement minimizes the number of broken bonds and lowers the energy (relative to the structure without rebonding) by $0.46 \mathrm{eV}$ per unit cell.

For total-energy calculations we used the GPAW code, ${ }^{21}$ a grid-based implementation of the projected augmented-wave method for all-electron calculations within the frozen core approximation. ${ }^{22}$ We used the local density approximation for the description of exchange and correlation. This approach has been shown to describe the structural properties of $\mathrm{SnS}$ with satisfactory accuracy compared to experiment and with much less computational effort compared to approaches that aim at high accuracy by improving the description of long-range dispersion forces, for instance using hybrid functionals. ${ }^{17,18}$ For $k$-space integration, a Monkhorst-Pack mesh was used and the position and overall shape of the main features of the density of states are converged with respect to the number of $k$-points, 4 or 8 along the in-plane reciprocal lattice directions.

\section{RESULTS}

After structural optimization of the unit cell, the calculated lattice constants of the orthorhombic phase of $\mathrm{SnS}$ are $\mathrm{a}_{0}=11.12 \AA, \mathrm{b}_{0}=3.95 \AA$, and $\mathrm{c}_{0}=4.24 \AA$, in reasonable agreement with experiment (the measured lattice constants at
$295 \mathrm{~K}$ are $\mathrm{a}_{0}=11.20 \AA, \mathrm{b}_{0}=3.99 \AA$, and $\mathrm{c}_{0}=4.33 \AA$ ). ${ }^{23}$ The distance between the closest two $\mathrm{Sn}$ atoms in two neighboring layers in the optimized cell, projected on the $a$-axis, is $2.62 \AA$. For each surface orientation, we calculate the surface energy, $\gamma$, defined as the energy required to create a unit surface from the bulk structure of $\mathrm{SnS}$ and obtained from

$$
\gamma=\frac{\mathrm{E}_{\mathrm{st}}^{N}-N \mathrm{E}_{\mathrm{b}}}{2 \mathrm{~A}}=\frac{N \mathrm{E}_{\mathrm{st}}^{N-1}-(N-1) \mathrm{E}_{\mathrm{st}}^{N}}{2 \mathrm{~A}}
$$

with $\mathrm{E}_{\mathrm{st}}^{N}$ the total energy of a stoichiometric slab consisting of $N$ atomic layers and surface area $\mathrm{A}$, and $\mathrm{E}_{\mathrm{b}}$ the total energy per atomic layer in the bulk. The factor of $1 / 2$ takes into consideration the two surfaces of the slab. When the extended bulk structure of $\mathrm{SnS}$ is used to calculate $\mathrm{E}_{\mathrm{b}}$ the surface energy diverges with increasing $N{ }^{24}$ Instead, we approximate the bulk energy with the incremental energy of the bulk-like interior of the slab using $\mathrm{E}_{\mathrm{b}}=\mathrm{E}_{\mathrm{st}}^{N}-\mathrm{E}_{\mathrm{st}}^{N-1}$ to ensure that the surface energy converges with the number of atomic layers and that the converged slab reliably describes both the surface and bulk regions of the material. Entropic and enthalpic contributions to the surface free energy are expected to be small at temperatures and pressures pertinent to experimental growth conditions of $\mathrm{SnS}$ thin films and they are neglected in our calculations. In this scheme, the lower the value for $\gamma$, the more stable the surface. The calculated surface energies are given in Table I. Because the bilayers in bulk $\mathrm{SnS}$ are arranged perpendicular to the long crystallographic axis ( $a$-axis) and are held together by weak forces in contrast to the strong intralayer bonds, the surface energy is minimized for the [100] surface, and is found to be $0.26 \mathrm{~J} / \mathrm{m}^{2}$. By contrast, intralayer bond breaking is naturally associated with higher surface energies, which vary in the narrow range between $0.57 \mathrm{~J} / \mathrm{m}^{2}$ and $0.63 \mathrm{~J} / \mathrm{m}^{2}$ for all other surfaces with the exception of the surface with [011] orientation for which $\gamma=0.38 \mathrm{~J} / \mathrm{m}^{2}$. For the most and least stable structures, the surfaces with [100] and [101] orientations respectively, the ratio of surface energies is 2.42 . This large ratio of surface energies may explain the observation of elongated grains in $\mathrm{SnS}$ samples at lower growth temperatures $\left(<120^{\circ} \mathrm{C}\right) .^{8,9}$

We next discuss trends in the electronic structure of the stoichiometric surfaces. In Fig. 2(a), we compare the density of states (DOS) for each surface orientation (shaded green) to that of the bulk (solid black line). There is an almost equal contribution from the $s$-orbital of $\mathrm{Sn}$ and $p$-orbital of $\mathrm{S}$ to the electronic states near the valence band maximum in the bulk band-structure. ${ }^{18}$ From this fact, we expect that surface formation by truncation of the bulk $\mathrm{SnS}$ will give rise to electronic states near the band-gap edges, as the intralayer

TABLE I. Surface energies, $\gamma$, for the low-index surfaces of layered SnS, and energy gain per $\mathrm{O}$ atom, $\Delta \epsilon$, for atomic substitution of the $\mathrm{S}$ by $\mathrm{O}$ at the surface sites.

\begin{tabular}{lcccccc}
\hline \hline Surface & {$[100]$} & {$[010]$} & {$[001]$} & {$[110]$} & {$[101]$} & {$[011]$} \\
\hline$\gamma\left(\mathrm{J} / \mathrm{m}^{2}\right)$ & 0.26 & 0.57 & 0.58 & 0.58 & 0.63 & 0.38 \\
$\Delta \epsilon(\mathrm{eV})$ & 1.65 & 1.92 & 1.49 & 2.31 & 2.14 & 2.27 \\
\hline \hline
\end{tabular}



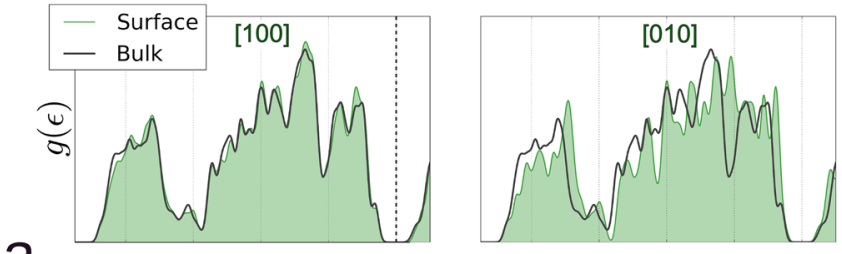

a
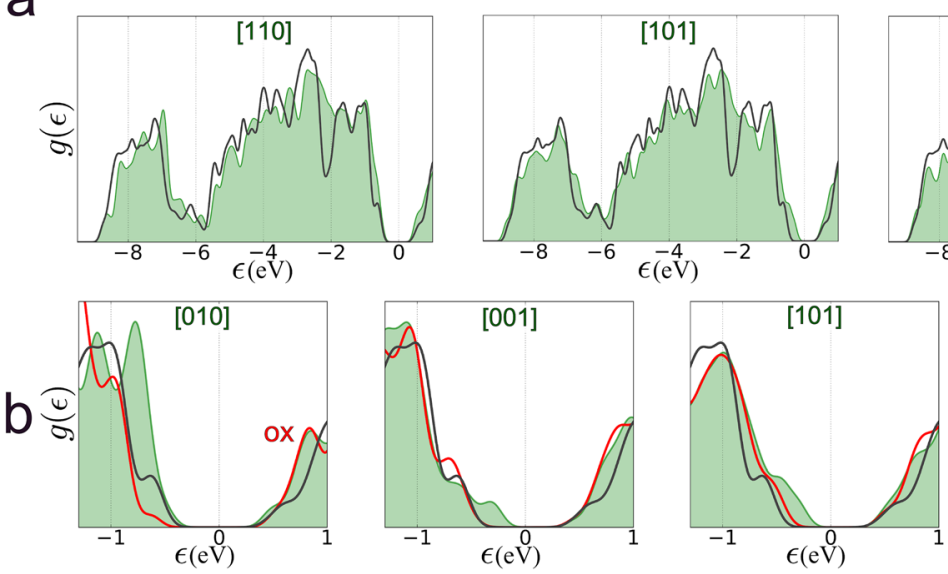
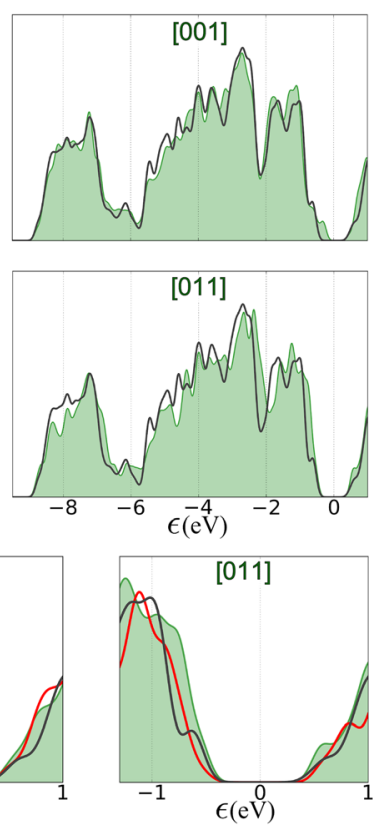

FIG. 2. (a, first and second rows) Density of states, $g(\epsilon)$, of bulk SnS (black solid line) and its stoichiometric (shaded green) low-index surfaces. The vertical dashed line marks the middle of the band gap of bulk SnS, defined as the zero of the energy scale. (b, third row) Close-up of the density of states near the band gap of bulk $\mathrm{SnS}$, and its stoichiometric and oxygen-containing ("ox," red solid line) surfaces for selected orientations. covalent bonds between Sn and S are broken. Such states, identified as additional peaks in the DOS of the surface compared to that of the bulk, are evident in all surface orientations examined except for the [100] orientation, which involves no bond breaking since it is parallel to the bilayer orientation in the bulk. For this particular orientation, the DOS is essentially identical to that of the bulk, indicating that exposing this surface makes no difference to the electronic properties of the material. This is also supported by the fact that relaxation has a negligible effect for the [100] surface: after optimization of the atomic positions of the bulk-terminated structure, the total energy per atom is reduced by less than $1 \mathrm{meV}$. The peaks related to surface states are particularly prominent for the orientations [010], [001], [101], [011], shown in greater detail in Fig. 2(b). In all these cases, the surface-related additional peaks appear both just below and just above the band-gap edges. To illustrate the nature of these states, we show in Fig. 3 the electron density of the surface-related peak of the occupied state at the center of the Brillouin zone which is closest to the Fermi level. The electron density corresponding to this state is localized at the top atomic layers and has the characteristic

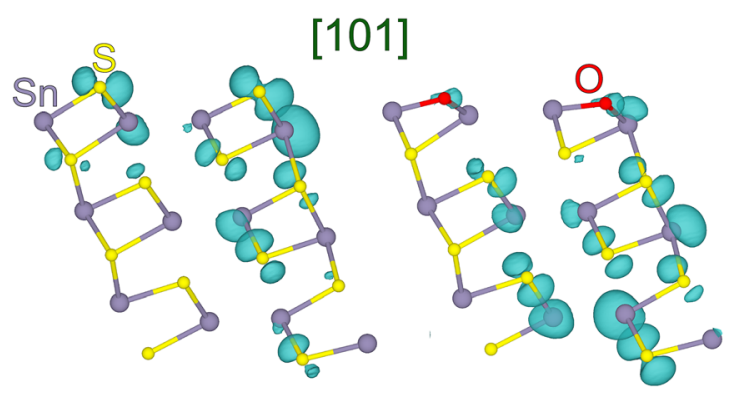

FIG. 3. Isosurfaces corresponding to $15 \%$ of the maximum electron density of the occupied state at the center of the Brillouin zone closest to the Fermi level: (left) the stoichiometric structure, and (right) the oxygen-containing structure of the $\mathrm{SnS}[101]$ surface. shape of a $p$-orbital near the exposed $\mathrm{S}$ atoms. Since the $\mathrm{S}$ atoms are three-fold bonded in the bulk with bond angles close to $90^{\circ}$, we can interpret the nature of this surface state as corresponding to a dangling bond. Such electronic states that appear near the top of the valence band would act as hole traps with detrimental effects on the efficiency of the PV cell.

In addition to the stoichiometric low-index surfaces, we consider model structures in which all the exposed $\mathrm{S}$ atoms are substituted by $\mathrm{O}$ atoms. This is relevant to experimental conditions of preparing the absorber layer, as discussed in more detail in Sec. IV. The effect of oxygen on the surface is to remove all the states associated with dangling bonds, as is evident from the DOS plots of Fig. 2(b). This effect is also shown in Fig. 3, where the substitution of $\mathrm{S}$ by $\mathrm{O}$ on the topmost atomic layer changes the character of the electronic state from a surface to a bulk state. These results can be rationalized by the relative position of the $\mathrm{S}$ and $\mathrm{O} p$-band. The center of the $\mathrm{O} p$-band, calculated from the first moment of the DOS projected on the $p$-orbitals of $\mathrm{O}$, is $0.86 \mathrm{eV}$ lower in energy compared to the $p$-band of $\mathrm{S}$ in the topmost atomic layer. Because the $\mathrm{O} p$-band lies lower in energy than the $\mathrm{S}$ $p$-band, substitution of the exposed $\mathrm{S}$ by $\mathrm{O}$ on the surface eliminates electronic states near the top of the valence band that are associated with the surface. Moreover, because oxygen has higher electronegativity (3.44 on the Pauling scale) than sulfur (2.58), more electron density is drawn to the surface $\mathrm{O}$ atoms. Using Bader analysis, ${ }^{25}$ we find the charge of the $\mathrm{O}$ atoms in the oxygen-containing [101] surface to be $1.2 e$, about $50 \%$ more than the charge of surface $\mathrm{S}$ atoms in the stoichiometric surface $(0.8 e)$. We conclude from these results that replacing $\mathrm{S}$ by $\mathrm{O}$ on surfaces or interfaces of $\mathrm{SnS}$, will reduce carrier recombination losses.

To quantify the effect of oxidation on the band structure of the model SnS surfaces, we calculate the minimum indirect and direct Kohn-Sham band gaps for the stoichiometric and oxygen-containing structures. As shown in Fig. 4, with 


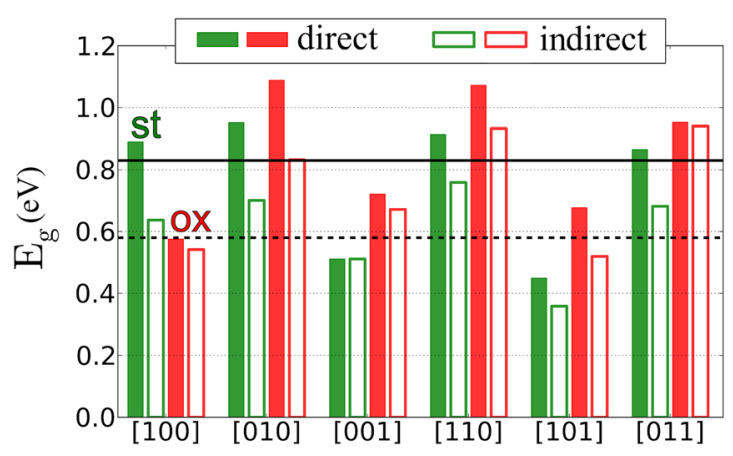

FIG. 4. Minimum Kohn-Sham band gap, $\mathrm{E}_{\mathrm{g}}$, for indirect (open bars) and direct (filled bars) transitions in stoichiometric ("st," green) and oxygen-containing ("ox," red) low-index surfaces of SnS. The horizontal black lines mark the calculated indirect (dashed) and direct (solid) band gaps of the bulk.

the exception of the surface with [100] orientation, the indirect and direct band gaps for each oxygen-containing surface are wider than the band gaps of the respective stoichiometric surfaces. The increase in the minimum Kohn-Sham band gap is in the range $0.09-0.22 \mathrm{eV}$ for indirect transitions and $0.13-0.26 \mathrm{eV}$ for direct transitions, with mean values of $0.18 \mathrm{eV}$ (31\% increase) and $0.16 \mathrm{eV}(27 \%)$, respectively. Within the framework of the local density approximation in density functional theory, the fundamental band gap of semiconductors and insulators is underestimated. In the case of bulk $\mathrm{SnS}$, the minimum Kohn-Sham band gap is $0.58 \mathrm{eV}$, underestimated by $0.49 \mathrm{eV}(46 \%)$ in comparison to experiment $(1.07 \mathrm{eV}) .{ }^{18}$ Furthermore, density functional theory is a ground-state theory in which excitonic effects are neglected in the calculation of optical transitions. Nevertheless, the passivation of surface states by oxygen is an effect due to changes in the chemical composition of the surface, which should not depend sensitively on the chosen approximation for exchange-correlation effects. We therefore expect that the trends we find in the density of states can give a reasonable estimate of the relative increase in measured optical band gaps for samples in which surface states are important.

\section{DISCUSSION AND CONCLUSIONS}

Our results for the effects of stoichiometric or oxygencontaining surfaces on the electronic properties of $\mathrm{SnS}$ have important implications for the behavior of the material under different conditions. Before addressing these implications, we need to establish whether it is possible or not to introduce $\mathrm{O}$ in the place of $\mathrm{S}$ in the surfaces of SnS. The energy gain per $\mathrm{O}$ atom is obtained from the expression $n \Delta \mathrm{E}=-\left[\mathrm{E}_{\mathrm{ox}}\right.$ $\left.-\mathrm{E}_{\mathrm{st}}-n\left(\mu_{\mathrm{O}}-\mu_{\mathrm{S}}\right)\right]$, where $\mathrm{E}_{\mathrm{st}}$ and $\mathrm{E}_{\mathrm{ox}}$ are the total energies of the stoichiometric and oxygen-containing slabs respectively, and $n$ is the number of atomic substitutions or, equivalently, number of $\mathrm{O}$ atoms in the resulting unit cell. ${ }^{26}$ The chemical potentials of the two species of atoms are given by $\mu_{\mathrm{S}}=-4.60 \mathrm{eV}$ obtained by using $\mathrm{S}_{8}$ molecules as the $\mathrm{S}$ reservoir, and $\mu_{\mathrm{O}}=-5.40 \mathrm{eV}$ obtained by using $\mathrm{O}_{2}$ molecules as the $\mathrm{O}$ reservoir. We find that for the various surfaces studied $\Delta \mathrm{E} / n$ is always positive, that is, the substitution of $\mathrm{S}$ by $\mathrm{O}$ is always exothermic. The values of $\Delta \mathrm{E} / n$ for the various surfaces studied here are given in Table I. Although these values are sensitive to the assumed reservoirs, they indicate a general trend of the oxygen-containing surfaces being more stable than their stoichiometric counterparts in an atmosphere rich in molecular oxygen. However, we find that diffusion of oxygen to the bulk is energetically unfavorable. For instance, switching an $\mathrm{O}$ atom in the topmost atomic layer of the [101] surface with a subsurface $S$ atom is inhibited by a minimum energy barrier of $0.33 \mathrm{eV}$, which suggests that an ultrathin oxidized surface layer could block the diffusion of sulfur out of the absorber layer. ${ }^{27}$

Having established that it is energetically favorable to modify the surface with $\mathrm{O}$, we can draw as a first conclusion that exposing the SnS surfaces to oxygen will eliminate the surface-related states near the band-gap edges (Fig. 2). In p-doped material, the surface-related states that lie near the top of the valence bands and are higher than the main peak in the bulk DOS (see Fig. 2(b)) would act as hole traps. These states are eliminated in oxygen-containing structures, which would lead to an improvement in the efficiency of the PV device.

The exothermic nature of the substitution of $\mathrm{S}$ by $\mathrm{O}$ on the $\mathrm{SnS}$ surfaces may have another beneficial effect, related to the presence of bulk defects. A typical strategy for tuning the properties of the absorber and buffer layers in the PV cell is annealing. This way, recombination of charge carriers in the absorber can be decreased as grains merge and grain boundary interfaces are reduced in size. Annealing can also passivate defects such as vacancies. For example, in the study of Park et al. ${ }^{13}$ post-deposition annealing in oxygen atmosphere was found to reduce the electron carrier concentration by decreasing the number of oxygen vacancies in the $\mathrm{Zn}(\mathrm{O}, \mathrm{S})$ buffer layer. According to Table I, during annealing in oxidizing atmosphere the surface of the SnS film will also be oxidized. Moreover, traces of oxygen may naturally be present in the material due to ambient contamination during film growth. We found that an oxidized surface layer could block diffusion of $S$ out of the absorber layer and inhibit the formation of S-vacancy defects which are in equilibrium with the surface. This could effectively reduce charge carrier trapping at $\mathrm{S}$ vacancy defects. Interpreting our oxygen-containing surfaces as structural models of ultrathin layers wherein $\mathrm{Sn}$ is oxidized, our findings suggest that growing an overlayer of tin oxide on the $\mathrm{SnS}$ film can enhance the conversion efficiency of the PV.

In conclusion, we find that low-index $\mathrm{SnS}$ surfaces have surface-related states near the band-gap edges, which would act as hole traps in p-doped material and be detrimental to the efficiency of the absorber. Electronic states that lie higher in energy than the main DOS peak of the bulk are eliminated by the substitution of surface $\mathrm{S}$ atoms by $\mathrm{O}$ atoms. This substitution is exothermic for all the surfaces we considered. In addition, the diffusion of oxygen toward the bulk is energetically inhibited. Moreover, the passivation of the surface by oxygen could also inhibit the formation of the dominant bulk point defect, S-vacancies. This would be beneficial to the efficiency of the photovoltaic cell by eliminating trapping of carriers by the defect-related states. Although optimization of the solar cell efficiency is ultimately a combinatorial 
challenge, these findings provide useful guidelines for improving the performance of thin film photovoltaic cells based on $\mathrm{SnS}$ absorbers by controlled growth of the $\mathrm{SnS}$ thin film.

\section{ACKNOWLEDGMENTS}

The authors wish to thank Katy Hartman and Prasert Sinsermsuksakul for their valuable comments and suggestions. This work was supported in part by the SEAS Dean's TomKat Fund for renewable energy research. Computations were performed on the Extreme Science and Engineering Discovery Environment (XSEDE), which is supported by National Science Foundation grant number OCI-1053575.

${ }^{1}$ N. Armaroli and V. Balzani, Angew. Chem. Int. Ed. 46, 52 (2007).

${ }^{2}$ S. Chu and A. Majumdar, Nature 488, 294 (2012).

${ }^{3}$ M. A. Green, K. Emery, Y. Hishikawa, W. Warta, and E. D. Dunlop, Prog. Photovolt. Res. Appl. 20, 12 (2012).

${ }^{4}$ W. Albers, C. Haas, and F. van der Maesen, J. Phys. Chem. Solids 15, 306 (1960).

${ }^{5}$ A. M. Elkorashy, Phys. Status Solidi B 159, 903 (1990).

${ }^{6}$ M. M. El-Nahass, H. M. Zeyada, M. S. Aziz, and N. A. El-Ghamaz, Opt. Mater. 20, 159 (2002).

${ }^{7}$ B. Ghosh, M. Das, P. Banerjee, and S. Das, Appl. Surf. Sci. 254, 6436 (2008).

${ }^{8}$ P. Sinsermsuksakul, J. Heo, W. Noh, A. S. Hock, and R. G. Gordon, Adv. Energy Mater. 1, 1116 (2011)

${ }^{9}$ K. Hartman, J. L. Johnson, M. I. Bertoni, D. Recht, M. J. Aziz, M. A. Scarpulla, and T. Buonassisi, Thin Solid Films 519, 7421 (2011).

${ }^{10}$ L. A. Burton, D. Colombara, R. D. Abellon, F. C. Grozema, L. M. Peter, T. J. Savenije, G. Dennler, and A. Walsh, Chem. Mater. 25, 4908 (2013).
${ }^{11}$ A. Wangperawong, S. M. Herron, R. R. Runser, C. Hägglund, J. T. Tanskanen, H.-B.-R. Lee, B. M. Clemens, and S. F. Bent, Appl. Phys. Lett. 103, 052105 (2013).

${ }^{12}$ P. Jain and P. Arun, Thin Solid Films 548, 241 (2013).

${ }^{13}$ H. H. Park, R. Heasley, and R. G. Gordon, Appl. Phys. Lett. 102, 132110 (2013).

${ }^{14}$ P. Sinsermsuksakul, K. Hartman, S. B. Kim, J. Heo, L. Sun, H. H. Park, R. Chakraborty, T. Buonassisi, and R. G. Gordon, Appl. Phys. Lett. 102, 053901 (2013).

${ }^{15}$ B. Ghosh, M. Das, P. Banerjee, and S. Das, Sol. Energy Mater. Sol. Cells 92, 1099 (2008)

${ }^{16}$ B. D. Malone and E. Kaxiras, Phys. Rev. B 87, 245312 (2013).

${ }^{17}$ L. Makinistian and E. A. Albanesi, Phys. Status Solidi B 246, 183 (2009).

${ }^{18}$ G. A. Tritsaris, B. D. Malone, and E. Kaxiras, J. Appl. Phys. 113, 233507 (2013).

${ }^{19}$ P. Zawadzki, L. L. Baranowski, H. Peng, E. S. Toberer, D. S. Ginley, W. Tumas, A. Zakutayev, and S. Lany, Appl. Phys. Lett. 103, 253902 (2013).

${ }^{20}$ W. Albers, C. Haas, H. J. Vink, and J. D. Wasscher, J. Appl. Phys. 32, 2220 (1961).

${ }^{21}$ J. Enkovaara, C. Rostgaard, J. J. Mortensen, J. Chen, M. Dułak, L. Ferrighi, J. Gavnholt, C. Glinsvad, V. Haikola, H. A. Hansen, H. H. Kristoffersen, M. Kuisma, A. H. Larsen, L. Lehtovaara, M. Ljungberg, O. Lopez-Acevedo, P. G. Moses, J. Ojanen, T. Olsen, V. Petzold, N. A. Romero, J. Stausholm-Møller, M. Strange, G. A. Tritsaris, M. Vanin, M. Walter, B. Hammer, H. Häkkinen, G. K. H. Madsen, R. M. Nieminen, J. K. Nørskov, M. Puska, T. T. Rantala, J. Schiøtz, K. S. Thygesen, and K. W. Jacobsen, J. Phys. Condens. Matter 22, 253202 (2010).

${ }^{22}$ P. E. Blöchl, Phys. Rev. B 50, 17953 (1994).

${ }^{23}$ A. R. H. F. Ettema, R. A. de Groot, C. Haas, and T. S. Turner, Phys. Rev. B 46, 7363 (1992).

${ }^{24}$ J. C. Boettger, Phys. Rev. B 49, 16798 (1994).

${ }^{25}$ W. Tang, E. Sanville, and G. Henkelman, J. Phys. Condensed Matter 21, 084204 (2009).

${ }^{26}$ G. X. Qian, R. M. Martin, and D. J. Chadi, Phys. Rev. B Condensed Matter 38, 7649 (1988).

${ }^{27}$ B. Ghosh, R. Bhattacharjee, P. Banerjee, and S. Das, Appl. Surf. Sci. 257, 3670 (2011). 